\title{
EL IMPACTO DEL ENTORNO MACROECONÓMICO EN EL DESARROLLO DE LA MIPYME: EL CASO DE MÉXICO
}

\author{
María Luisa Saavedra García*, Miriam Edith Saavedra García
}

\begin{abstract}
Saavedra-García M.L., Saavedra-García M.E. El impacto del entorno macroeconómico en el desarrollo de la MiPyME: El caso de México. Hitos de Ciencias Económico Administrativas 2015;21 (59): 53-66
\end{abstract}

RESUMEN

El objetivo de este trabajo consiste en determinar de qué manera el entorno macroeconómico, enmarcado bajo el concepto del nivel macro de competitividad sistémica, influye en el desarrollo de la MiPyME en México.

Este trabajo se desarrolló en tres partes; la primera, se conceptualiza y describen los indicadores del nivel macro de competitividad, así también se da cuenta de las investigaciones antecedentes que han intentado establecer una relación entre las variables del entorno y la competitividad de las empresas; la segunda, se realiza la descripción del entorno macroeconómico de la MiPyME en México, mostrando la evolución de las variables más importantes que afectan al desempeño empresarial; por último, se presenta un análisis FODA en el que se determina con respecto al entorno macroeconómico de que manera este, afecta o beneficia al desarrollo de la MiPyME.
Saavedra-García M.L., Saavedra-García M.E. El impacto del entorno macroeconómico en el desarrollo de la MiPyME: El caso de México. Hitos de Ciencias Económico Administrativas 2015;21 (59): 53-66

\section{ABSTRACT}

The aim of this study is to determine how the macroeconomic environment, framed under the concept of systemic competitiveness at the macro level, influences the development of the SME in Mexico.

This work took place in three parts the first is conceptualized and described the macro-level indicators of competitiveness, and realizes that background investigations have attempted to establish a relationship between environmental variables and competitiveness of enterprises. In the second part, it is described in the macroeconomic environment of the SME in Mexico, showing the evolution of the most important variables affecting business performance. Finally, we present a SWOT analysis that is determined with respect to the macroeconomic environment in which way this affects or benefits the development of SMEs.

Palabras clave: Competitividad. Nivel macro. MiPyME.

Key words: Competitiveness. Macro level. SME. Policy. Políticas.

DIRECCIÓN PARA RECIBIR CORRESPONDENCIA: Correo electrónico: maluisasaavedra@yahoo.com

* Investigadora de la Facultad de Contaduría y Administración de la Universidad Nacional Autónoma de México.

** Investigadora de Tiempo Completo de la Universidad del Mar, Oaxaca, México. 


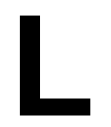

as pequeñas empresas pueden ayudar a reducir la pobreza puesto que generan gran cantidad de empleo, niveles adecuados de calidad de empleo y bajo costo de bienes y servicios utilizados por los pobres (Vanderberg, 2007). Sin embargo, no son ajenas a las amenazas del entorno que en determinado momento pueden limitar su desempeño (Benacek, 1995).

En México como en muchos otros países de América Latina, Asia, y Estados Unidos, la micro pequeña y mediana empresa o MiPyME ${ }^{1}$ conforma la inmensa mayoría del universo empresarial. De ahí la importancia estratégica que éstas adquieren o deberían tener en términos del desarrollo económico nacional, regional o local pues al año 2009 se cuenta con más de 5 millones de empresas (productores agrícolas, ganaderos, acuicultores, mineros, artesanos, manufacturas, comercios, servicios turísticos y culturales) las cuales se distribuyen de acuerdo con el tamaño ${ }^{2}$ como se muestra en en la tabla 1.

Como se puede apreciar en la tabla 1, la importancia de la MiPyME es latente, si consideran que generan el $78.5 \%$ del empleo y aportan el $52 \%$ al PIB nacional.

\section{Tabla 1}

\section{Distribución de Empresas Mexicanas}

\begin{tabular}{lcccc}
\hline Empresas & $\begin{array}{c}\text { No. de } \\
\text { empresas }\end{array}$ & $\begin{array}{c}\text { \% del total } \\
\text { de empresas }\end{array}$ & $\begin{array}{c}\text { \% de generación } \\
\text { de empleos }\end{array}$ & $\begin{array}{c}\text { \% de aportación } \\
\text { al PIB }\end{array}$ \\
\hline Micro & $4^{\prime} 877,070$ & $94.80 \%$ & $45.60 \%$ & $15.50 \%$ \\
Pequeñas & 214,956 & $4.18 \%$ & $23.80 \%$ & $14.50 \%$ \\
Medianas & 42,415 & $0.82 \%$ & $9.10 \%$ & $22.00 \%$ \\
MIPYME & $5 ' 134,441$ & $99.80 \%$ & $78.50 \%$ & $52.00 \%$ \\
Grandes & 9,615 & $0.20 \%$ & $21.50 \%$ & $48.00 \%$ \\
Total & $5 ' 144,056$ & $100.00 \%$ & $100.00 \%$ & $100.00 \%$ \\
\hline
\end{tabular}

Fuente: Elaboración propia en base a Censos Económicos INEGI 2009.
El objetivo de este trabajo consiste en determinar de qué manera el entorno macroeconómico, enmarcado bajo el concepto del nivel macro de competitividad sistémica, influye en el desarrollo de la MiPyME en México.

Este trabajo se desarrolló en tres partes; en la primera, se conceptualiza y describen los indicadores del nivel macro de competitividad, así también se da cuenta de las investigaciones antecedentes que han intentado establecer una relación entre las variables del entorno y la competitividad de las empresas; en la segunda, se realiza la descripción del entorno macroeconómico de la MiPyME en México, mostrando la evolución de las variables más importantes que afectan al desempeño empresarial; por último, se presenta un análisis FODA en el que se determina con respecto al entorno macroeconómico de que manera este, afecta o beneficia al desarrollo de la MiPyME.

\section{El nivel de competitividad Macro}

Esser, et al. (1994), respecto de la competitividad macro, han señalado que: lo más importante es que el contexto macroeconómico permanezca estable (es decir, que la inflación, el déficit presupuestario, los tipos de cambio y la deuda externa sean controlables y, además, que las reglas de juego no se alteren permanentemente; esa es la única forma de generar seguridad para la inversión), y que la política macro emita señales claras e inequívocas para dar a entender a las empresas que están en la obligación de aproximarse al nivel de eficiencia habitual en el ámbito internacional. A tal efecto puede ser útil la política comercial, si ésta va reduciendo a pasos previsibles

${ }^{1}$ La MiPyME en México de acuerdo con la publicación del 30.06.2009, en el diario oficial de la federación se clasifica de la siguiente manera: a) Microempresa es la que tiene hasta 10 empleados y un nivel de ventas de hasta 4 millones de pesos anuales, b) Pequeña empresa del sector comercio es la que tiene de 11 a 30 empleados y un nivel de ventas de hasta 100 millones de pesos anuales, c) Pequeña empresa de los sectores servicio e industria, es la que tiene de 11 a 50 empleados y un nivel de ventas de hasta 100 millones de pesos anuales, d) Mediana empresa del sector comercio es la que tiene de 31 a 100 empleados y un nivel de ventas de hasta 250 millones de pesos, e) Mediana empresa de los sector servicios tiene de 51 a 100 empleados y un nivel de ventas de hasta 250 millones de pesos, f) Mediana empresa industrial es la que tiene de 51 a 250 empleados y un nivel de ventas de hasta 250 millones de pesos.

${ }^{2}$ Esta clasificación se realizó de acuerdo con el número de empleados. 
las barreras a la importación. La política de competencia puede jugar por su parte un rol significativo para impedir la creación de situaciones monopólicas.

La estabilización en el nivel macro es una premisa necesaria, pero no suficiente para hacer sustentable el desarrollo de la competitividad. Reviste gran importancia la implementación de las políticas meso. Su formulación, no obstante, tiene pocas perspectivas de éxito mientras no se emprenda la estabilización del contexto macro. Por ejemplo, la política tecnológica no alcanzará su objetivo de fortalecer la competencia tecnológica de las empresas mientras los propios empresarios no procuren la competitividad. Las barreras selectivas a la importación amparan el fortalecimiento de las industrias únicamente cuando las empresas aprovechan el tiempo para hacerse competitivas. Según Esser, et al. (1994) y Hernández (2001), los factores determinantes de competitividad sistémica a nivel Macro son:

La política monetaria procura un marco estable (baja tasa inflacionaria), sin obstaculizar la inversión con tasas de interés demasiado elevadas. Donde el mercado de moneda y de capitales está poco desarrollado, una política monetaria orientada a la estabilidad se encuentra con fuertes limitaciones, dado que la aplicación de medidas habituales como: racionamiento crediticio, la concesión selectiva de créditos y fijación arbitraria de tasas de interés, provoca a su vez nuevas distorsiones en los mercados de moneda y capitales sin poder modificar el volumen crediticio en la medida deseada.

La política presupuestaria persigue mantener el déficit bajo control para garantizar la consistencia macro. Los déficits presupuestarios persistentemente elevados que se financian mediante la creación de dinero generan altas tasas de inflación, que distorsionan los precios y por consiguiente, la operatividad del mecanismo de mercado. El déficit en la balanza de pagos, cuando se mantiene alto durante un largo tiempo debido a un tipo de cambio sobrevaluado, genera también efectos inflacionarios. Provocando una elevada deuda externa que sumada al servicio de la misma debilita la inversión nacional, obstaculiza la importación de bienes de capital poniendo así en peligro las bases de crecimiento de la economía nacional.

La política fiscal estimula la productividad de la inversión. Un buen sistema tributario está escalonado con justicia y transparencia, siendo de carácter progresivo.

La política de competencia contrarresta la formación de monopolios y cárteles, así como el abuso de poder de mercado.

La política cambiaria está concebida para impedir que las exportaciones encuentren obstáculos y las importaciones necesarias se encarezcan demasiado. Las experiencias pasadas han demostrado que los tipos de cambio, cuando están muy sobrevaluados generan déficits en la balanza, sobre todo porque dificulta la exportación de manufacturas y se facilita la importación de las mismas.

La política comercial fomenta una integración activa al mercado mundial. Las empresas necesitan percibir señales claras provenientes de la política comercial que las motiven a dirigir sus estrategias hacia el mercado mundial.

Los factores señalados anteriormente, tienen impacto en los diferentes de agregación política como se muestra en la tabla 2.

\section{Tabla 2}

Factores determinantes de competitividad Sistémica a diferentes niveles de agregación política
(nivel macro)
\begin{tabular}{lccc} 
Supranacional & Nacional & Regional & Local \\
\hline $\begin{array}{l}\text { Flujos internacionales } \\
\text { de capital }\end{array}$ & $\begin{array}{c}\text { Marcos macroeconómicos } \\
\text { (sistemas tributario, } \\
\text { capacidad de financiamiento) }\end{array}$ & $\begin{array}{c}\text { Política fiscal sólida. } \\
\text { Capacidad de } \\
\text { inversión publica. }\end{array}$ & $\begin{array}{c}\text { Política fiscal sólida. } \\
\text { Capacidad de inversión } \\
\text { publica. }\end{array}$ \\
$\begin{array}{c}\text { Calidad de vida, medio y } \\
\text { contexto oportuno. }\end{array}$
\end{tabular}

Fuente: Hernández (2001). 
Como puede observarse en la tabla 1, los factores de competitividad en el nivel macro tienen impacto en el nivel supranacional a través de los flujos internacionales de capital, en el nivel nacional a través de los marcos macroeconómicos, en el nivel regional a través de una política fiscal sólida y la capacidad para realizar inversión y en el nivel local a través de una política fiscal sólida, capacidad de inversión pública, calidad de vida. La articulación de estos factores entre estos cuatro niveles es determinante para alcanzar la competitividad en el nivel. Macro.

\section{La relación entre las variables del entorno y la competitividad de las empresas}

Carree, at al. (2002), intentaron explicar la interrelación entre el número de emprendedores y desarrollo económico, se encontró evidencia de que existe un equilibrio a largo plazo entre el desarrollo económico y los emprendedores.

Smallbone y Welter (20019) consideran que las diversas formas en que el Estado puede influir en la naturaleza y el ritmo de desarrollo de las MiPyME, son:

1. Puede influir a través de su política macroeconómica. En cualquier país, un entorno macroeconómico estable, hace que sea más fácil para a las empresas planificar, sobre todo con respecto al momento de la inversión, la expansión y asumir nuevas iniciativas.

2. El segundo modo en que puede influir a través de una legislación diferenciada para empresas de diferente tamaño.

3. Una tercera forma en que puede influir es diseñando programas de apoyo a la MiPyME que la ayuden a salvar las limitaciones inherentes al tamaño (recursos internos limitados).

4. Una cuarta forma en que el Estado puede influir es a través de su apoyo para el desarrollo de las instituciones del mercado tales como la infraestructura de apoyo a las empresas, bancos y otros intermediarios financieros.

5. Por último, el papel que juega el Estado en relación con el desarrollo de las MiPyME, dada la influencia que tiene al brindar apoyo para la creación de empresas y el fomento del espíritu empresarial en la sociedad. Claramente, esta es una influencia a largo plazo y es afectada por muchos factores, incluyendo las características del sistema educativo (en todos los niveles), que incluye tanto el plan de estudios y métodos de enseñanza. Sin embargo, también es influenciada por la postura que el gobierno tiene con respecto a animar a las personas a iniciar y desarrollar sus propios negocios y por el comportamiento de políticos y funcionarios del gobierno en sus relaciones con las pequeñas empresas.

Choi y Phan (2006), realizaron un estudio acerca de cómo la política macroeconómica y tecnológica de un país influyen en la dinámica de la formación de nuevas empresas; se busco la relación entre el número de empresas nuevas de los años a 1993 (por cada mil habitantes), y los indicadores macroeconómicos y tecnológicos como: inversión per cápita investigación y desarrollo, número de patentes per cápita, concentración económica, competencia política, desempleo y sindicalismo. Los resultados sugieren que las inversiones en I + $\mathrm{D}^{3}$, patentes, concentración económica, la política pro-competencia, y la movilidad laboral son áreas importantes en que la política del gobierno puede influir para la formación de nuevas empresas.

De otro lado, Bibu, et al. (2009), realizaron una investigación que tuvo como objetivo determinar la influencia de factores internos y externos sobre la competitividad de las empresas, los resultados les permitieron identificar factores que impulsan el continuo crecimiento de la MiPyME como son: la mejora de las capacidades de gestión, la mejora de los canales de distribución, la reducción de costos, la modernización de la tecnología, el crecimiento de la capacidad de producción, la mejora en la motivación de los empleados.

Así también, identificaron factores positivos de influencia en el medio ambiente externo que fueron: crecimiento de la demanda por sus productos en los mercados locales y extranjeros; modernización de la infraestructura, oportunidades para el financiamiento de las inversiones, simplificación de acceso a los préstamos, tasas de interés atractivas, mejor calidad de materias primas, métodos y tecnologías modernas de producción, aumento poder de compra de la

\footnotetext{
3 Investigación y desarrollo.
} 
población, modificaciones de las normas, las políticas gubernamentales. Al mismo tiempo, identificaron factores, que pueden tener un impacto negativo en el desarrollo empresarial: el aumento de los precios de las materias primas y los servicios públicos, el crecimiento de la competencia. Llegando a la conclusión que las instituciones del gobierno central y locales juegan un papel muy importante en las actividades de la empresa.

Asimismo, Roper y Hart (2005) en otra investigación, encontraron que las políticas de apoyo a las MiPyME tienen un efecto positivo en el empleo, una mejora en los procesos de la empresa aunque este último no pudo ser reflejado en el rendimiento.

Con respecto a la carga tributaria, Carroll, Holtz-Eakin, Riden y Rosen (2000) en un estudio empírico comprobaron que cuando más alta era la tasa del impuesto de las personas físicas con actividad empresarial, más baja era la tasa de crecimiento de las empresas, realizando con base en este resultado realizaron una propuesta acerca de la implementación de una tasa diferenciada de impuestos para este tipo de empresas. Por lo que promover el crecimiento del empleo, a través de políticas que impulsen el fomento a las pequeñas empresas, pueden tener ventajas y desventajas, como vemos a continuación (Congressional Budget Office, 2012):

$>$ El costo de cumplir con las regulaciones federales, puede ser especialmente gravoso para las pequeñas empresas. Por ejemplo, el cumplimiento de leyes federales, tales como las destinadas a evitar la discriminación o a reducir la contaminación a menudo requiere que las empresas desarrollen sistemas o procedimientos internos que tienen un costo fijo por empresa, además de los costos que varían con el tamaño de la firma. En ese caso, cumplir con la regulación en pequeñas empresas será más costoso de lograr que en las empresas grandes. Por lo tanto, se deben implementar normas modificadas para las pequeñas empresas o eximirlas de algunas regulaciones, y de este modo promover el crecimiento del empleo.

$>$ La desventaja de las políticas que favorecen a las pequeñas empresas, es que tales políticas pueden desalentar a las empresas para aumentar en tamaño al perder este tratamiento preferente. Por otra parte, eximir a las pequeñas empresas a partir de ciertos reglamentos o normas modificadas aplicadas a las mismas, puede originar que persistan algunos problemas, tales como discriminación o contaminación.

Algunas reducciones de impuestos patronales estimulan el empleo logrando que las pequeñas empresas contraten trabajadores adicionales. Sin embargo, dado que los trabajos proporcionados por las empresas pequeñas son menos «durables», el beneficio económico de cada puesto de trabajo subvencionado es menor si el impuesto reducido a la nómina se limita a las pequeñas empresas. Lo que implica que el costoefectividad de la política relativa a la reducción de impuestos, no es relevante, por lo que muchos gobiernos no lo implementan.

Las políticas federales tienen influencia sobre la capacidad de las empresas para desarrollarse y ser eficientes, dado que son las que determinan los impuestos que las empresas pagan, la disponibilidad de crédito, las normas con las que debe cumplir y otros factores. Así por ejemplo, las políticas fiscales, afectan significativamente a las decisiones sobre todo si desea iniciar un nuevo negocio o ampliar uno ya existente. Sobre todo si las tasas impositivas se aplican a todas las empresas por igual sin importar el tamaño.

Por consiguiente, las reformas en el entorno regulatorio que buscan reducir la burocracia y los largos procedimientos en el registro de nuevas empresas son beneficiosas para el crecimiento y sustento de nuevas MiPyME (Olawale y Garwe, 2010).

En este sentido, Hualde (1998) señala que las políticas públicas en la actualidad tratan de rectificar el rumbo pues en épocas pasadas tenían prioridad las grandes empresas dado que se infería que eran las que impulsaban el desarrollo de los países, sin embargo en la actualidad las MiPyME han demostrado ser las impulsoras del desarrollo, de este modo se ha reconocido que se encuentran en desventaja frente a las empresas grandes y se le trata de brindar apoyo a través de subsidios al financiamiento, al empleo.

Así también, Morand (2003) afirma que una forma en la que los gobiernos han intentado insertar a las MiPyME en sus economías es a través de las compras del 
gobierno, en las que pueden tener dos tipos de participación: 1) Contratar directamente con una entidad pública, y 2) Participar como subcontratista de un contratista principal. Sin embargo, a pesar de esfuerzos que realizan los gobiernos para impulsar esta participación en Europa la participación directa apenas alcanza al $25 \%$ de las compras, mientras que en Chile esta participación es de $45 \%$ y en México esta apenas alcanza el 20\% (Saavedra, 2011).

Acompañadas de un marco adecuado de políticas, estas empresas y en particular sus segmentos potencialmente más dinámicos, pueden erigirse en una palanca de transformación de las economías latinoamericanas, a través de los siguientes procesos (OCDE-CEPAL, 2012):

Contribuir a aumentar la productividad. Mediante la introducción de cambios tecnológicos y organizativos. De esa forma, las MiPyME podrían actuar como agentes que fomenten el cambio estructural, ayudando a la creación y difusión de innovaciones y el desarrollo de nuevos mercados. Asimismo, la entrada de nuevas empresas podría aumentar la competencia, incorporar nuevos modelos de negocios que desafían los tradicionales generando una turbulencia saludable («destrucción creativa» en términos schumpeterianos), que conlleva a la búsqueda constante de un uso más productivo de los recursos y contribuye al aumento de la productividad agregada de una economía (Altenburg y Eckhardt, 2006).

Complementar las economías de escala de las grandes empresas. Con las ventajas en flexibilidad, las MiPyME pueden lograr menores costos de transacción como consecuencia del contacto cercano con clientes y la toma de decisiones más rápidas. Las MiPyMEs, en particular, quedarían en condiciones de acceder a mercados diversificados (a través de la exportación o ventas a grandes cadenas comerciales) y cadenas globales de valor, beneficiándose de las transferencias de tecnología (Dini y Stumpo, 2002).

Desarrollar un papel importante en la creación de aglomeraciones productivas. Concebidas como una colaboración entre agentes económicos e instituciones en el plano local, y cuyo propósito es la generación de un beneficio competitivo. Esta interacción permite a las empresas involucradas lograr niveles de producción más elevados, incorporar tecnologías con mayor facilidad, acelerar procesos de aprendizaje y, en definitiva, conseguir un nivel de eficiencia colectiva que no sería alcanzable por una firma individual (Ferraro y Stumpo, 2010).

Contribuir a la inclusión social al aumentar los ingresos de las microempresas y reducir su vulnerabilidad. Muchas microempresas latinoamericanas se han creado como una estrategia de supervivencia debido a la falta de dinamismo de actividades intensivas en el uso de mano de obra. Con frecuencia, estos segmentos de la población no son pobres, pero integran sectores vulnerables. Su absorción por sectores dinámicos no está garantizada, porque tomaría bastante tiempo y no necesariamente las personas tienen las capacidades requeridas. Algunas políticas de apoyo a las microempresas (como intervenciones multidimensionales relacionadas a las microfinanzas) son eficaces y eficientes para brindar herramientas que permitan elevar los ingresos y conferirles estabilidad en un contexto de redes de protección social reducida e incompleta.

Así pues, las pequeñas empresas que identifican un alto grado de turbulencia deben buscar fuentes estables de financiamiento, deben flexibilizar su estructura operativa y considerar la diversificación para reducir riesgos. En cambio aquellas que cuentan con un bajo grado de turbulencia pueden arriesgarse en el mercado, tomar financiamiento de corto plazo y realizar cambios en el largo plazo en su estructura operativa.

\section{El entorno macroeconómico de la MiPyME en México}

La situación económica del país se caracteriza por bajo crecimiento económico, altas tasas de interés y comisiones bancarias que inhiben la inversión productiva. Lo anterior ha contribuido a que las MiPyMES que representan el $99.80 \%$ de los establecimientos económicos para el año 2008, recurran preferentemente al crédito de proveedores (Saavedra y Hernández, 2008). Resulta pertinente señalar que la MiPyMES se caracterizan por ser empresas familiares, con problemas de financiamiento en el corto, mediano y largo plazo, además de dificultades de acceso a la innovación tecnológica, así como la falta de recursos humanos capacitados para realizar estudios de mercado, financieros y técnicos que le permitan 
incursionar exitosamente en el ámbito internacional (Saavedra, et al. 2007).

Figura 1

Producto interno bruto (\% de crecimiento)

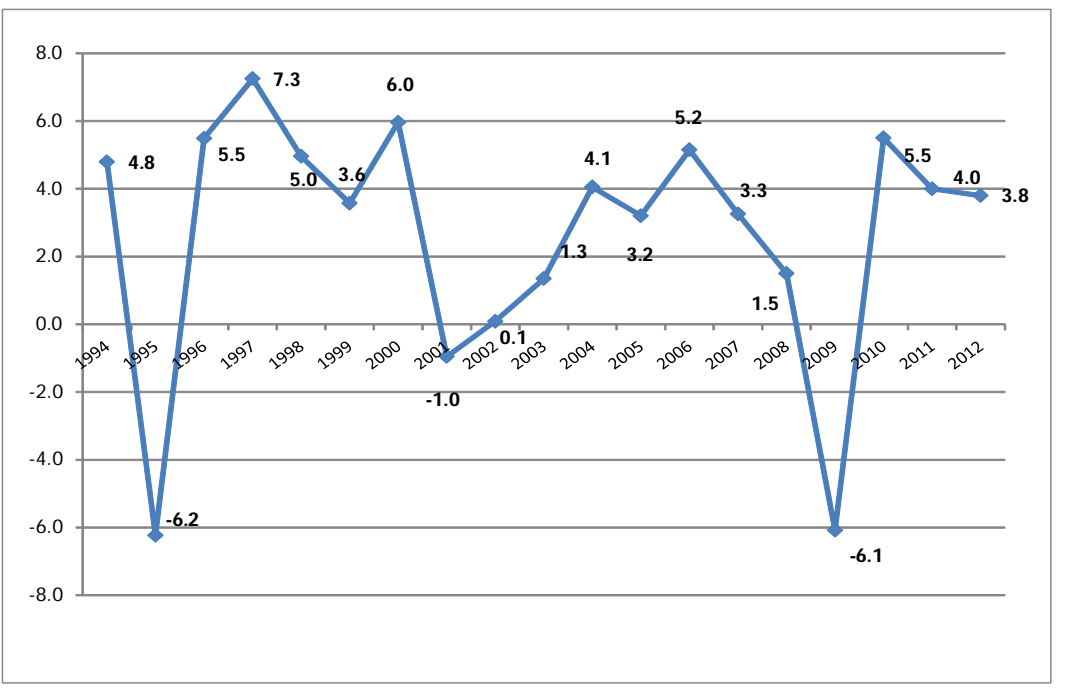

Fuente: Banco de México

A continuación, se presenta una descripción y análisis de los principales indicadores del entorno Macroeconómico.

\section{El crecimiento económico}

El indicador más importante del crecimiento económico de México es el producto interno bruto, como puede verse, en la figura 1, en el período 1994-2012, el crecimiento promedio fue de $2.6 \%$ anual, mismo que resulta insuficiente para superar los grandes problemas de desempleo, marginación y pobreza. Además, como México ha sufrido el impacto de tres grandes crisis económicas 1995, 2001 y 2009, mostrando con esto la gran dependencia que existe del sector externo sobre todo de Estados Unidos, ya que alrededor del $80 \%$ de las importaciones y exportaciones se realizan con este país (Blecker, 2009).

Así también, uno de los aspectos que ha impedido el crecimiento en México es la baja competitividad, los estudios realizados por Ros (2004), señalan que estos aspectos son: competitividad cambiaria, desarrollo institucional y entorno empresarial. En cuanto a la competitividad medida de acuerdo al Foro económico mundial México se encuentra en el lugar 66 de 139 países, por debajo de Chile y Puerto Rico que se encuentran en el lugar 41. Los escollos de la competitividad mexicana, según los expertos del Foro, siguen siendo la ineficiencia de un gobierno burocrático, la corrupción, el difícil acceso al financiamiento, el crimen, la violencia y las regulaciones restrictivas a la iniciativa privada.

\section{Política Monetaria}

En 2001 el Banco de México adoptó un esquema de objetivos de inflación como marco conceptual para conducir su política monetaria. De manera específica, el Banco de México se ha propuesto alcanzar, en el mediano plazo, una tasa de inflación anual del Índice Nacional de Precios al Consumidor (INPC) del $3 \%$.

No obstante, cabe destacar que aun cuando la política monetaria se conduzca apegada a la consecución de dicha meta, en la práctica, debido a los múltiples factores que inciden sobre la inflación, existe cierto margen de incertidumbre sobre el cumplimiento exacto y puntual de la misma. Por ello, se ha establecido un intervalo de variabilidad de más/menos un punto porcentual alrededor de la meta del 3 por ciento (Banco de México, 2003). Cabe destacar, que el Banco de México ha determinado en los últimos años, una política monetaria restrictiva $a^{4}$, en un esfuerzo por contener la presión inflacionaria.

\section{Evolución de la inflación}

Como puede apreciarse, en la figura 2 la evolución de la inflación ha sido positiva al pasar de un 52\% en 1994 a un $3.57 \%$ en 2012 , esto debido a la política de estabilidad de precios aplicada por el Banco de México (con el fin de promover el crecimiento económico a largo plazo (Avendaño y Vázquez 2011).

Es necesario señalar que el corto $^{5}$ ha sido un instrumento muy útil para Banco de México en la conducción de la política monetaria, porque ha permitido

${ }^{4}$ Política de Bancos Centrales diseñada para detener la inflación por medio de la reducción de reservas de los bancos comerciales repercutiendo en la disminución de la oferta monetaria. 


\section{Figura 2}

Inflación (INPC variación anual)

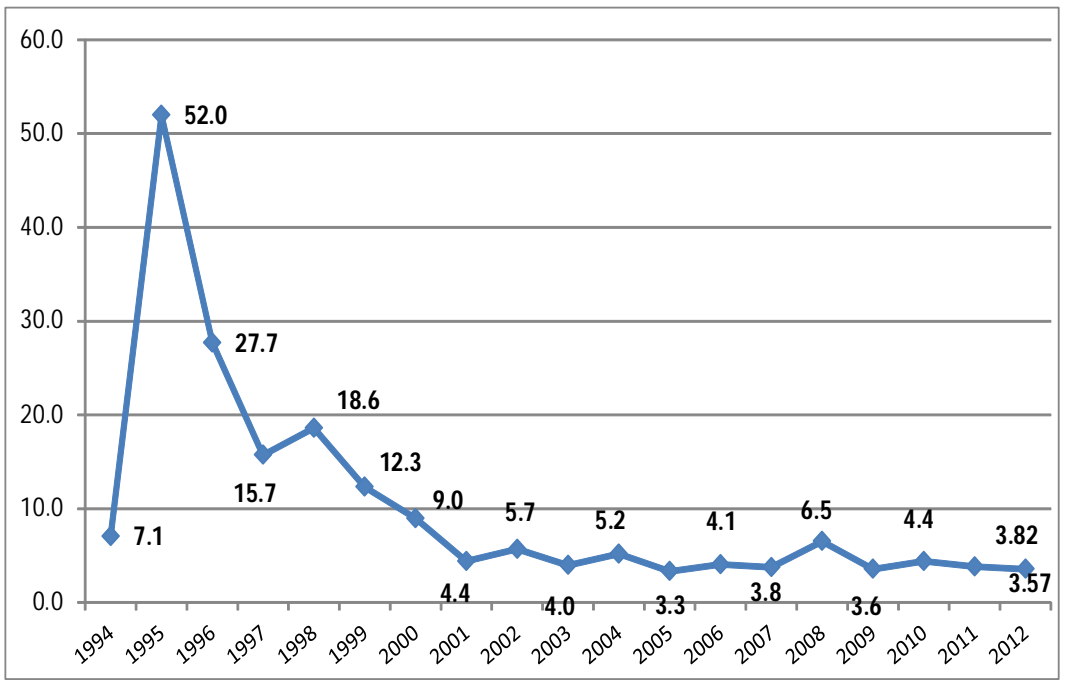

Fuente: Banco de México

que los choques a los mercados se distribuyan y se absorban mediante movimientos tanto en las tasas de interés de corto plazo como en el tipo de cambio. Es el propio mercado quien ha distribuido la intensidad de estos choques en ambas variables, y en las condiciones de volatilidad en que ha vivido la economía en épocas anteriores y recientes.

\section{Evolución de la tasa de interés}

La tasa de interés ha mostrado una disminución importante de $48.4 \%$ en 1996 hasta alcanzar $4.26 \%$ en 2012 (ver figura 2), como resultado de la decisión del Banco de México de mantener estas tasas en congruencia con el comportamiento de la inflación y el tipo de cambio (Banco de México, 2011). Además de la recuperación de la economía internacional aunque no se descartan riesgos futuros.

Sin embargo, los expertos señalan que cuando la tasa de interés se utiliza para contrarrestar aumentos en el valor del tipo de cambio se da una contradicción

5 El corto o Sistema de saldos acumulados, es un instrumento de política monetaria que utiliza el Banco de México para abatir la inflación. Consiste en dejar menos liquidez en el sistema (menor circulación de billetes y monedas) que elimina presiones inflacionarias. interna en términos de la fragilidad financiera de las empresas (Avendaño y Vázquez, 2011). Pues, cuando el tipo de cambio se deprecia, y en consecuencia aumenta el valor en moneda nacional de los pasivos denominados en moneda extranjera de las empresas, el Banco Central aumenta la tasa de interés a fin de mantener el control de la inflación, pero esto produce dos efectos: el aumento en el riesgo del prestamista y del prestatario y la modificación adversa en la estructura financiera de las empresas desincentivando de este modo la inversión figura 3.

Es de notar que a pesar de la baja en la tasa de referencia en este caso los CETES (Certificados de la tesorería de la federación), las tasas de interés para el otorgamiento de préstamos bancarios a la MiPyME es muy elevada, debido al alto costo de la intermediación financiera que maneja la banca mexicana y a los altos riesgos que representan las MiPyME (Lecuona, 2009).

\section{Política Presupuestaria}

La disciplina en el manejo de las finanzas públicas ha permitido tener un déficit moderado y una deuda decreciente.

Figura 3.

Certificado de la tesorería de la federación (CETES 28 días)

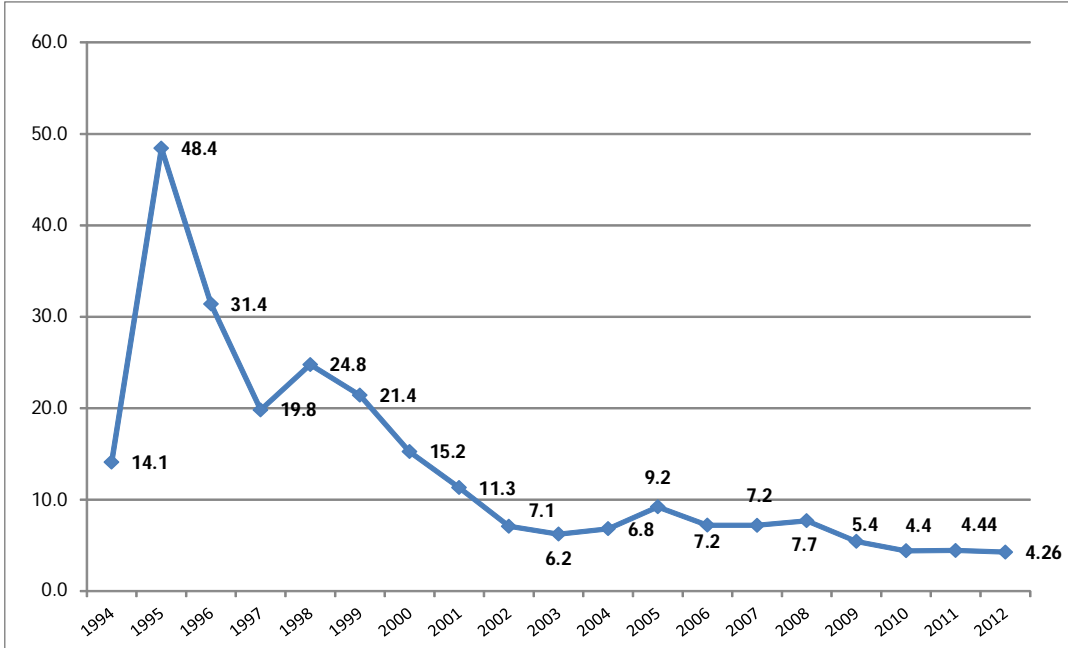

Fuente: Banco de México. 


\section{Deuda interna y externa}

En los últimos años, el gobierno ha implementado la política de sustituir deuda externa por interna, esto se puede observar en la gráfica 4 , donde puede verse que en el año 2004 la deuda externa representaba el 24.6\% del PIB, mientras que la deuda interna era tan sólo el 10.8\%. Al año 2012, la deuda externa representa el $10.48 \%$ del PIB, mientras que la deuda interna es el $22.1 \%$ del PIB. Es en el año 2000 cuando Moody's otorga el grado de inversión a la deuda mexicana, lo que permitió disminuir los costos de la misma, esto debido también la reconfiguración de la estructura de la deuda, enfocándose a la deuda interna con la colocación de instrumentos financieros en moneda nacional y a largo plazo (CEFP, 2009).

Las mejoras en la estructura de la deuda pública han contribuido a reducir de forma significativa la vulnerabilidad de la economía mexicana a cambios en el entorno interno o externo (CEFP, 2002; CEFP, 2006). Cabe destacar que el nivel de deuda total que alcanzó México en el año 2010 fue de $32.5 \%$ del PIB, mientras que otros países como por ejemplo Estados Unidos era en ese año cerca del $80 \%$, por lo que se considera que la deuda mexicana es manejable (SHCP, 2011). (ver figura 4).

\section{Figura 4}

Deuda interna y externa (\% de PIB)

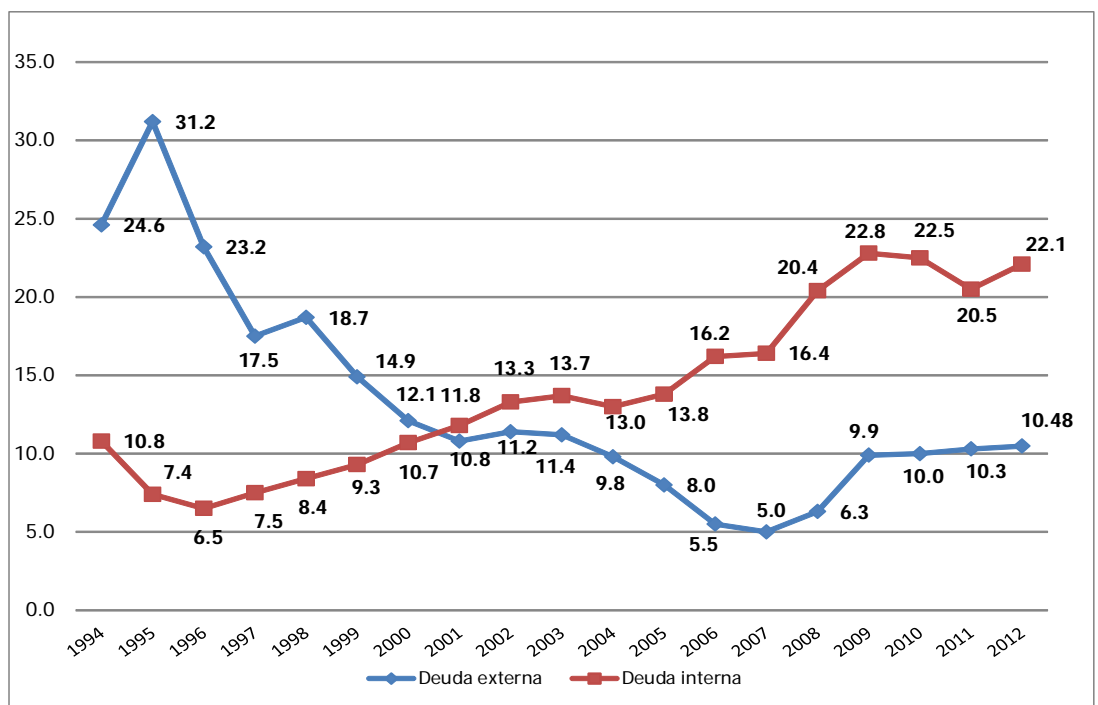

Fuente: Secretaría de Hacienda y Crédito Público.

\section{Figura 5}

Balance público (\% del PIB)

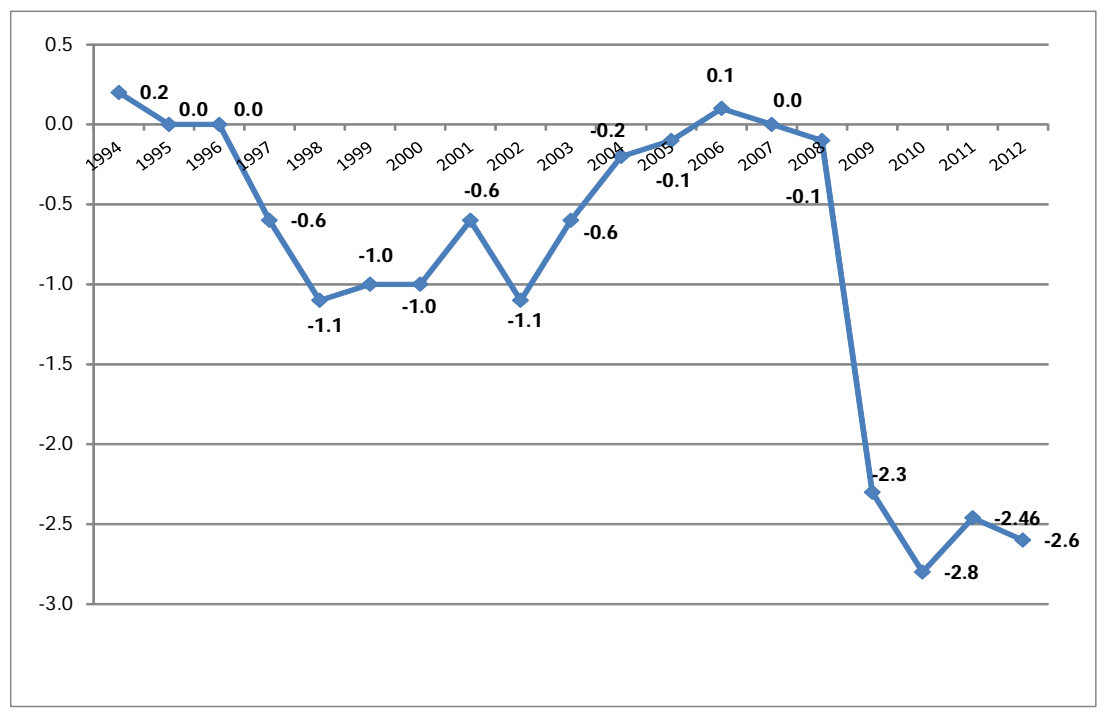

Generalmente, la evolución de la deuda pública está ligada al resultado que muestra el balance público tradicional (o económico), mismo que muestra el cambio en la posición financiera neta del sector público que se obtiene de restar al ingreso corriente y de capital los gastos de la misma naturaleza. Dicho resultado debe ser equivalente al financiamiento que se deriva del endeudamiento más el uso o acumulación de disponibilidades.

Cómo puede apreciarse en la figura 5, la tendencia del balance público desde 1994 al año 2012 es deficitario, dado que los ingresos corrientes del sector público resultan insuficientes para sufragar sus gastos de allí que siempre tengan que ser solventados con endeudamiento.

La Organización para la Cooperación y el Desarrollo Económico (OECD, 2011), recomienda que México debe tomar en cuenta los siguientes puntos para futuras iniciativas de reforma presupuestaria. A) Desarrollar un enfoque a largo plazo, b) Incluir rendición de cuentas en la formulación del presupuesto, c) Comprometer al congreso en la discusión del presupuesto, d) Fomentar que el sector público se enfoque en los resultados. 


\section{Política Fiscal}

Tradicionalmente las finanzas públicas mexicanas han mostrado fuerte problemas estructurales. Existe un ingreso escaso derivado de una carga fiscal muy baja (comparada con estándares internacionales), concentración de las fuentes de ingresos y pequeña base tributaria, entre otras características. El ingreso derivado de la recaudación fiscal tan sólo representa aproximadamente el $11 \%$ del PIB, uno de los más bajos, si lo comparamos con los otros miembros de la OCDE. Por otro lado, el escaso ingreso repercute en un gasto público que resulta insuficiente, con presiones en un futuro cercano que se deberán enfrentar en materia de pensiones, mayor gasto en capital y menores ingresos petroleros. Habría que agregar las distorsiones que contiene el sistema tributario mexicano mediante tratamientos especiales, subsidios, exenciones, y deducciones en el Impuesto Sobre la Renta, y productos con tasa cero o exentos como en el caso del Impuesto al Valor Agregado (Werner y Ursúa, 2005).

Régimen fiscal aplicado a las pequeñas empresas Para el gobierno en turno la facilidad en el cumplimiento del pago de los impuestos, es esencial para que los nuevos negocios que se van creando, se incorporen a la formalidad, con todas las ventajas que esto representa para el país (IMCP, 2014). El Sistema de Administración Tributaria (SAT), es el organismo encargado de recaudar los impuestos en México. Uno de los mecanismos para que se simplificara el pago de impuestos, se denominaba Régimen de Pequeños Contribuyentes (REPECOS). Los que se inscribían en este régimen, estaban exentos de llevar una contabilidad formal, así como de conservar comprobantes de sus proveedores, ni presentar declaración anual. La única obligación formal, era la de pagar una cuota bimestral, en ningún caso mayor al $5 \%$ de los ingresos totales en el período.

Actualmente, el SAT está poniendo en marcha un nuevo mecanismo para que los micros y pequeños empresarios paguen sus impuestos, este se denomina Régimen de Incorporación Fiscal (RIF), que sustituye al Régimen de Pequeños Contribuyentes ${ }^{6}$ (REPECOS). Todos los pequeños empresarios que tributaban como REPECOS pasaron de manera automática, a partir de 2014 al RIF y cualquier negocio nuevo tiene que inscribirse en este nuevo régimen. Cualquier microempresario que no tenga ingresos anuales mayores a dos millones de pesos mexicanos $(150,000$ dólares americanos aproximadamente) puede formar parte del mismo, en el que se podrá tributar durante 10 años (SHCP-SAT, 2014). Una vez que trascurra este lapso de tiempo, el contribuyente pasará al régimen general aplicable a todos los contribuyentes, por lo que se puede considerar a este nuevo régimen como temporal.

En este nuevo régimen los principales beneficios que se ofrecen son: descuentos en el pago de los impuestos a cargo de la empresa (el primer año y por única vez no se pagaran impuestos), servicios de salud para el pequeño empresario y su familia, pensión para el retiro, crédito para vivienda y financiamiento para el negocio. La comunicación oficial enfatiza los beneficios de la formalidad, con la intención que los negocios informales se inscriban al RIF. Para obtener estos beneficios, es indispensable un cumplimiento cuidadoso de las obligaciones fiscales a cargo de la empresa, de no hacerlo así, automáticamente el contribuyente pasaría a un régimen general sin exenciones ni descuentos, es decir se pierden los beneficios.

Para el cumplimiento de las obligaciones, en este régimen se está implementando una plataforma informática, en la que el contribuyente registrará sus ingresos y sus gastos cada dos meses. Asimismo, se pueden generar facturas electrónicas a través de esta misma aplicación. Para las personas que no cuenten con internet es decir, que vivan en localidades rurales que de acuerdo al criterio del Instituto Nacional de Estadística y Geografía (INEGI) cuenten con menos de 2,500 habitantes y en las que la totalidad de los habitantes censados hayan confirmado la ausencia del servicio de internet, se ha implementado un número de teléfono gratuito, de tal manera que puedan realizar sus declaraciones y registro por este medio.

\footnotetext{
${ }^{6}$ México se aplicaba el Régimen de Pequeños Contribuyentes, conocido como REPECOS, en el que se integran el Impuesto a la Renta y el Impuesto sobre Valor Agregado. A él podían adherirse las personas físicas que realizaban actividades empresariales, siempre que vendieran o prestaran servicios al público, cuando los ingresos propios de su actividad más los intereses obtenidos en el año anterior no superaran los dos millones de pesos (U\$D186.133) (BID, 2008). Establecía una cuota única integrada por una cuota fija correspondiente al Impuesto al Valor Agregado, considerando un importe de IVA mensual de $\$ 100$, y otra resultante de aplicar una alí cuota del $2 \%$ sobre los ingresos brutos anuales.
} 
En teoría al menos, la facilidad para cumplir con las obligaciones fiscales a través de plataformas informáticas en internet más los beneficios que ofrece el RIF, incrementaría notablemente la base tributaria. Sin embargo, la baja capacidad administrativa de muchos micros y pequeños empresarios, hace que aún resulte complejo. Algunos especialistas afirman también, que es demasiado ambicioso, pretender generar todo por internet, cuando aún la penetración de las tecnologías de información no han permeado a una gran parte de la población mexicana.

La Cámara de Comercio, Servicios y Turismo en Pequeño de la ciudad de México (CANACOPE), aseguró que aún hay dificultades y desconfianza para registrarse en el sistema, sobre todo por el hecho de ser un esquema temporal, en el que después de 10 años pasarán a tributar al igual que una gran empresa. Habrá que ver si este nuevo régimen puede atraer a aquellos que hoy realizan una actividad empresarial en la informalidad y sin contribuir al gasto público o resulta contraproducente y se incrementa el número de empresarios que opten por los negocios en la informalidad con las desventajas que esto supone.

\section{Ingresos tributarios como porcentaje del PIB}

En México los ingresos tributarios están muy por debajo de sus principales socios comerciales pues mientras recauda en promedio del año 1990 a 2009, un $8.5 \%$ del PIB, Estados Unidos logra una recaudación de $28 \%$ en promedio en este mismo período y Canadá alcanza un promedio aún mucho más elevado 35\% (CEFP, 2006; SHCP, 2011). Es de mencionar que los expertos señalan que existe una relación directa entre los ingresos tributarios y el crecimiento económico por lo que el principal desafío para México sería sentar las bases para un crecimiento sólido y sostenido (Cedillo, 2007).

\section{Política de competencia}

En junio de 1993, entra en vigor la Ley Federal de Competencia Económica (LFCE). Esta ley creó a la Comisión Federal de Competencia (CFC) como organismo autónomo única responsable de su aplicación. Como un órgano desconcentrado de la Secretaría de Economía (SE), encargado de aplicar la Ley Federal de Competencia Económica (LFCE), que entró en vigor el 22 de junio de 1993. La CFC logró la aprobación de una serie de reformas a la LFCE que entraron en vigor el 28 de junio de 2006, las cuales simplifican y mejoran diversos procedimientos asociados con la aplicación de la LFCE. Entre estos se encuentran: (i) la reducción de plazos en el análisis de concentraciones y la aclaración de los agentes sujetos a la ley; (ii) la ampliación de las facultades de la CFC; y (iii) el establecimiento de opiniones vinculativas de la CFC, que son de carácter obligatorio y se emiten a entidades de la administración pública federal respecto de programas y políticas que pudieran tener efectos contrarios al proceso de competencia y libre concurrencia.

La CFC investiga las denuncias y casos y los tramita ante el Poder Judicial Federal. Sin embargo, la elevada carga de casos del poder judicial aunado a la falta de conocimientos especializados sobre el tema, impiden que jueces y magistrados revisen de forma sustantiva los casos de competencia. Esto se ha traducido en un lento avance de los casos, siendo uno de los efectos más ilustrativos de esta situación la proliferación de juicios de amparo que se otorgan con base en fallas de procedimientos y cuyo propósito es dilatar la acción de la CFC para así prolongar las rentas monopólicas de algunos importantes grupos económicos del país. En los últimos cinco años, por ejemplo, han ingresado 277 juicios de amparo contra actos definitivos de la CFC y 315 contra actos procesales (BID, 2006).

Las MiPyME son particularmente vulnerables a las prácticas anticompetitivas que pudieran llevar a cabo diversos agentes económicos, incluyendo prácticas de abuso de poder de mercado, así como la colusión entre competidores, particularmente en licitaciones públicas. En buena medida, muchos de estos comportamientos tienen origen en regulaciones municipales y estatales que violan la LFCE, y en el desconocimiento de conductas ilegales por parte de autoridades estatales y municipales así como de asociaciones, cámaras y gremios locales.

\section{Política Cambiaria}

En los primeros dos meses de 2009, con un entorno internacional adverso, se observó un deterioro en los indicadores de riesgo país de las economías emergentes, así como depreciaciones importantes de sus monedas y aumentos en la volatilidad en los mercados cambiarios. En el caso de México, la reducción de los ingresos petroleros y las perspectivas de menores ingresos públicos no petroleros como 
resultado de la contracción en la actividad económica, generaron incertidumbre sobre los márgenes de maniobra de las finanzas públicas para 2009 y, en particular, para 2010. Adicionalmente, en el contexto de menores ingresos de divisas y condiciones de astringencia prevalecientes en los mercados financieros internacionales, a principios de 2009 surgió la percepción entre los inversionistas de que México podría tener problemas para financiar el moderado incremento proyectado para 2009 en el déficit en la cuenta corriente. Ello provocó un episodio de elevada incertidumbre en el mercado cambiario, y repuntes importantes en los indicadores de riesgo soberano del país.

\section{Evolución del tipo de cambio}

Ante el entorno internacional adverso antes señalado, el tipo de cambio registró una depreciación considerable. Dada esta circunstancia, los precios de las mercancías, relativos a los precios de los servicios tienden a ajustarse en la misma dirección que los cambios que se observan en el tipo de cambio. En particular, una depreciación duradera del tipo de cambio eventualmente conduce a un aumento en el precio relativo de las mercancías.

Los analistas señalan que el peso se encuentra sobrevaluado, esto desde luego afecta al sector exportador y beneficia la entrada de productos del extranjero a precios más bajos que los nacionales, restándole de este modo competitividad al productor mexicano.

\section{Política Comercial}

La balanza comercial en el período 1994-2012, muestra un déficit promedio de $\mathrm{U} \$ 5,626$ millones de dólares, esto se debe principalmente a la estructura productiva de México que depende de insumos importados ${ }^{7}$, siendo esto un elemento importante para la restricción del crecimiento, ya que aún a pesar de la dinámica exportadora de los últimos años su estructura productiva no le permite financiar estos déficits (Loria, 2001).

${ }^{7}$ Esto debido al bajo desarrollo tecnológico de la industria mexicana.

\section{Tabla 3}

Análisis FODA entorno Macro de la MiPyME

El entorno macroeconómico tiene una afectación sobre la MiPyME, altas tasas de inflación y elevadas tasas de interés desincentivan la inversión y por ende generan desempleo, a continuación se presenta un análisis FODA, (ver tabla 3).

\section{CONCLUSIONES}

A pesar de estabilidad que muestran los indicadores de la economía mexicana, la misma no se encuentra ajena a los efectos adversos de la economía en el entorno externo dada la alta interdependencia económica y financiera de economías más desarrolladas como la de Estados Unidos.

Bajo este contexto, el resultado de este trabajo nos permite determinar las fortalezas, debilidades, amenazas y oportunidades del entorno macro que enfrenta la MiPyME mexicana, entre las principales fortalezas se encuentra un nivel de inflación que permanece bajo dado los constantes controles que ejerce el gobierno para evitar que la misma se descontrole y desincentive de este modo las inversiones, lo anterior a su vez se encuentra aunado a las bajas tasas de interés pasivas y de fondeo bancario, siendo que las tasas activas guiadas por la ley de oferta y demanda son las más altas de la región y no le permiten al empresario de este sector contar con financiamiento externo. Como consecuencia de esto, entre las principales debilidades se encuentra el difícil acceso a

\section{Análisis FODA del entorno Macro de la MiPyME}

\begin{tabular}{|c|c|}
\hline Fortalezas & Debilidades \\
\hline $\begin{array}{l}\text { - Baja inflación } \\
\text { - Bajas tasas de interés } \\
\text { - Nivel de deuda pública aceptable } \\
\text { - Régimen fiscal diferenciado para los } \\
\text { primeros años de vida }\end{array}$ & $\begin{array}{l}\text { - Gobierno burocrático } \\
\text { - Difícil acceso a fuentes de financiamiento } \\
\text { - Enfoque presupuestario de corto plazo } \\
\text { - Falta de protección contra los monopolios } \\
\text { - Volatilidad en el tipo de cambio que eleva } \\
\text { el precio de los insumos } \\
\text { - Peso sobrevaluado que no fomenta las } \\
\text { exportaciones } \\
\text { - Bajo desarrollo de tecnología propia }\end{array}$ \\
\hline Amenazas & Oportunidades \\
\hline $\begin{array}{l}\text {-Bajo crecimiento económico } \\
\text {-Dependencia del sector externo (EU } \\
\text { y Canadá) } \\
\text {-Bajo nivel de competitividad } \\
\text {-Bajo nivel de ingresos tributarios }\end{array}$ & $\begin{array}{l}\text {-Adquisición de insumos y mercancías } \\
\text { importadas a un costo bajo }\end{array}$ \\
\hline
\end{tabular}

Fuente: Elaboración propia. 
fuentes de financiamiento por parte del empresario de este sector, situación que representa el segundo problema más importante de la MiPyME en Latinoamérica, después de la corrupción, otra debilidad importante se encuentra en el bajo desarrollo de Tecnología propia, situación que le impide a al MiPyME ser competitiva en este aspecto, pues al no contar con herramientas para mejorar sus procesos, le resulta imposible elevar su productividad.

Por otra parte, en lo que se refiere a las amenazas una de las más importantes es el bajo crecimiento económico, influenciado por un entorno mundial que no ha sido favorable para el país, pues la baja de los precios del petróleo en los últimos años ha mermado los recursos que el estado podría destinar a inversión productiva con el consecuente efecto multiplicador para la economía que entre otros aspectos se traduce en generación de empleos. Aunado a esto, se encuentra la recesión económica en Estados Unidos, país del que dependemos económicamente pues del total de lo que vendemos en el exterior por lo menos el $80 \%$ lo hacemos a nuestro vecino del norte. En cuanto a las oportunidades encontradas, la principal es que al mantenerse un peso sobrevaluado es posible que el empresario pueda adquirir insumos y mercancías importadas a un costo más bajo, permitiéndole esto vender o producir a precios competitivos.

Es así como, el empresario MiPyME tiene que tomar decisiones que implican un alto grado de riesgo económico, principalmente cuando él mismo dirige sus negocios dentro de un contexto o situación de crisis en el mercado. La historia económica demuestra que la aparición de estas crisis en los mercados es, de hecho, muy frecuente y multifacética. En la toma de decisiones, la experiencia del empresario, pasa a ser un elemento clave, puesto que los negocios están expuestos a operar en diferentes escenarios económicosociales a lo largo de sus vidas activas.

Por último, el empresario MiPyME debe comprender como las variables del entorno económico afectan a su empresa y prepararse para tomar las decisiones que atenúen el impacto adverso que el mismo pueda tener.

\section{REFERENCIAS}

Altenburg, T. y Eckhardt, G. (2006). Productivity enhancement and equitable development: challenges for SME development. Vienna: United Nations Industrial Development Organization.

Avendaño, V. y Vázquez, J. (2011). Inestabilidad financiera y política monetaria en México, 19902008. Investigación Económica, LXX(275), enero marzo, 2011, pp. 63-92.

Banco de México (2003). Informe sobre la inflación octubre-diciembre 200 y programa monetario para 2003. Enero 2003.

Banco de México (2011). Boletín de prensa, 4 de marzo de 2011.

Banham, H. (2010). External environmental analysis for small and medium enterprises (SMEs). Journal of business \& economic research, 8(10) 19-25.

Benacek, V. (1995). Problems and environment of small businesses in the Czech Republic. Small business in the Czech Republic. (7) 437-450.

Bibu, N., Stefea, P., Sala, D. (2009). External and internal environment influencesiness on SME competitiveness from the western area of Romania. Scientific Review Paper. 6 (2) 41-52.

BID (2006). Programa de apertura de mercados a PYMES por medio de la Política de competencia. México: Banco Interamericano de Desarrollo.

BID (2008). Recomendaciones y mejores prácticas para la tributación de PYMES en Latinoamérica. México: Banco Interamericano de Desarrollo.

Blecker, R. (2009). External Shocks, Structural Change, and Economic Growth in Mexico, 19792007. World Development, 37(7), pp. 1274-1284.

Búrdalo, O.; Serra, J., Medina, J. y Stefanut, G. (2008). La Política Económica en México (20002007).

Carree, M., Van, Ac., Thurik, R. \& Wennekers, S. (2002). Economic Development and Business Ownership: An Analysis Using Data of 23 OECD Countries in the Period 1976-1996. Small Business Economics. 19: 271-290.

Carroll, R., Holtz-Eakin, D., Rider, M., y Rosen, H. (2000). Personal income taxes and the growth of small firmes. National Bureau of Economic Research. Working paper 7980.

Cedillo, E. (2007). Crítica a la estructura tributaria actual en México. Contaduría y Administración , 223, 113-134.

CEFP (2002). Breves Comentarios sobre la Evolución de la Deuda Pública Mexicana y de otros países.Cámara de Diputados. Documento 0016/ 2002. Centro de estudios de las Finanzas Públicas, México. 
CEFP (2006).Evolución de la deuda pública en México y sus principales componentes. Cámara de Diputados. Documento 0039/2006. Centro de estudios de las Finanzas Públicas, México.

CEFP (2009). Indicadores de ingresos tributarios en países seleccionados. Cámara de Diputados. Documento 0071/2009. Centro de estudios de las Finanzas Públicas, México.

Choi, Y. \& Phan, P. (2006). Influences of Economic and Technology Policy on the Dynamics of New Firm Formation. Small Business Economics. 26: 493-503.

Congressional Budget Office (CBO), (2012). Small firms, employment, and federal policy. USA: Congress.

Dini, M. y Stumpo, G. (2002). Análisis de la política de fomento a las pequeñas y medianas empresas en Chile. Serie Desarrollo Productivo 136. CEPAL, Santiago de Chile.

Esser, K., Wolfgang, H., Dirk, M. y Meyer-Stamer, J. (1994). Competitividad sistémica. Competitividad internacional de las empresas y políticas requeridas. Berlín: Instituto Alemán de desarrollo.

Ferraro, C. y Stumpo, G. (2010). Políticas de apoyo a las PYMES en América Latina. Entre avances innovadores y desafíos institucionales. Santiago de Chile: Naciones Unidas-Cepal.

Hernández, R. (2001). Elementos de competitividad Sistémica de las Pequeñas y Medianas Empresas (MIPYME) del Istmo Centroamericano. México: Naciones Unidas-CEPAL.

Hualde, A. (1998). PYMEs y desarrollo regional: La importancia de los enfoques europeos y sus limitaciones. Perfiles Latinoamericanos, 13, 199227.

IMCP. (2014). Instituto Mexicano de Contadores Públicos. Fisco Actualidades. Nuevo Régimen de Incorporación Fiscal de las Personas Físicas. Febrero 2014. Disponible en: http:// imcp.org.mx/wp-content/uploads/2014/02/ Fisco-febrero-2014-03.pdf, Consultado del 05 de marzo de 2014.

Lecuona, R. (2009). El financiamiento a las Pymes en México: Una experiencia reciente. Economía, UNAM. 6(17), 69-91.

Loria, E. (2001). El desequilibrio comercial en México ¿o porqué ahora no podemos crecer? algunos elementos conceptuales. Momento económico (113) 16-21.

Morand, P. (2003). SME and public procurement policy. Review of Economic Design, 8, 301-318.

OCDE-CEPAL (2012). Perspectivas económicas para América Latina 2013. Políticas de PYMES para el cambio estructural. Chile: Autor.
OECD (2010). Revenue Statistic 1965-2009: 2010 edition.

OECD (2011). Economic, Politicy, Reforms 2011: Going for Growth.

Olawale, y Garwe, (2010). Obstacles to the growth of new SMEs in South Africa: A principal component analysis approach. African Journal of Business Management, 4(5), 729-738.

Programme Job Creation and Enterprise Development Department International Labour Office - Geneva.

Roper, S. \& Hart, M. (2005). Small Firm Growth and Public Policy in the UK: what exactly are the connections? Aston Business School Research Papers. RP 0504.

Ros, J. (2004). El crecimiento económico en México y Centroamérica: desempeño reciente y perspectivas. Serie estudios y perspectivas 18 . México: CEPAL.

Saavedra, M. (2011). Chile una potencia en compras de gobierno. El economista, suplemento el empresario, 28 de marzo, 2011, 8.

Saavedra, M. et al. (2007). Perfil Financiero y administrativo de las Pequeñas Empresas en el Estado de Hidalgo. México: UAEH.

Saavedra, M. y Hernández Y. (2008). Perfil financiero de las pequeñas empresas: caso Estado de Hidalgo, México. Contaduría Universidad de Antioquia, 53, 173-196.

SHCP (2011). Política Fiscal 2000-2010. México: Secretaría de Hacienda y Crédito Público.

SHCP-SAT. (2014) Secretaría de Hacienda y Crédito Público y Servicio de Administración Tributaria. Folleto Crezcamos Juntos. Disponible en: http://www2.sat.gob.mx/CrezcamosJuntos/ 06.htm. Consultado el 01 de abril de 2014.

Smallbone, D. y Welter, F. (2001). The role of government in SME development in the transition economies of central and Eastern and the newly independent states. Conference proceedings the fourth International Conference on Enterprise in Transition. Croacia.

Vandenberg, P. (2007). Poverty reduction through small enterprises Emerging consensus, unresolved issues and ILO activities. Small Enterprise Development.

Páginas de internet:

http://www.banxico.org.mx

http://www.shcp.gob.mx

http://www.inegi.gob.mx 